\title{
Knowledge of Retinoblastoma by Healthcare Professionals in Sub-saharan Africa: Survey Performed in the Republic of Côte d'Ivoire and the Democratic Republic of the Congo
}

\author{
Lukamba Mbuli Robert ${ }^{1, *}$, Monga Bondo Ben ${ }^{2}$, Yao Atteby ${ }^{3}$, Kabesha Amani Théo ${ }^{4}$, \\ Budiongo Nzazi Aléine ${ }^{5}$, Bey Pierre ${ }^{6}$, Chenge Borasisi Gabrielle ${ }^{7}$, Desjardins Laurence ${ }^{8}$, \\ Savignoni Alexia ${ }^{9}$, Berete-Coulibaly Rokia ${ }^{10}$, Couitchere Line ${ }^{11}$, Luboya Numbi Oscar ${ }^{12}$, \\ Doz François ${ }^{13}$, Mwembo Tambwe Albert ${ }^{14}$
}

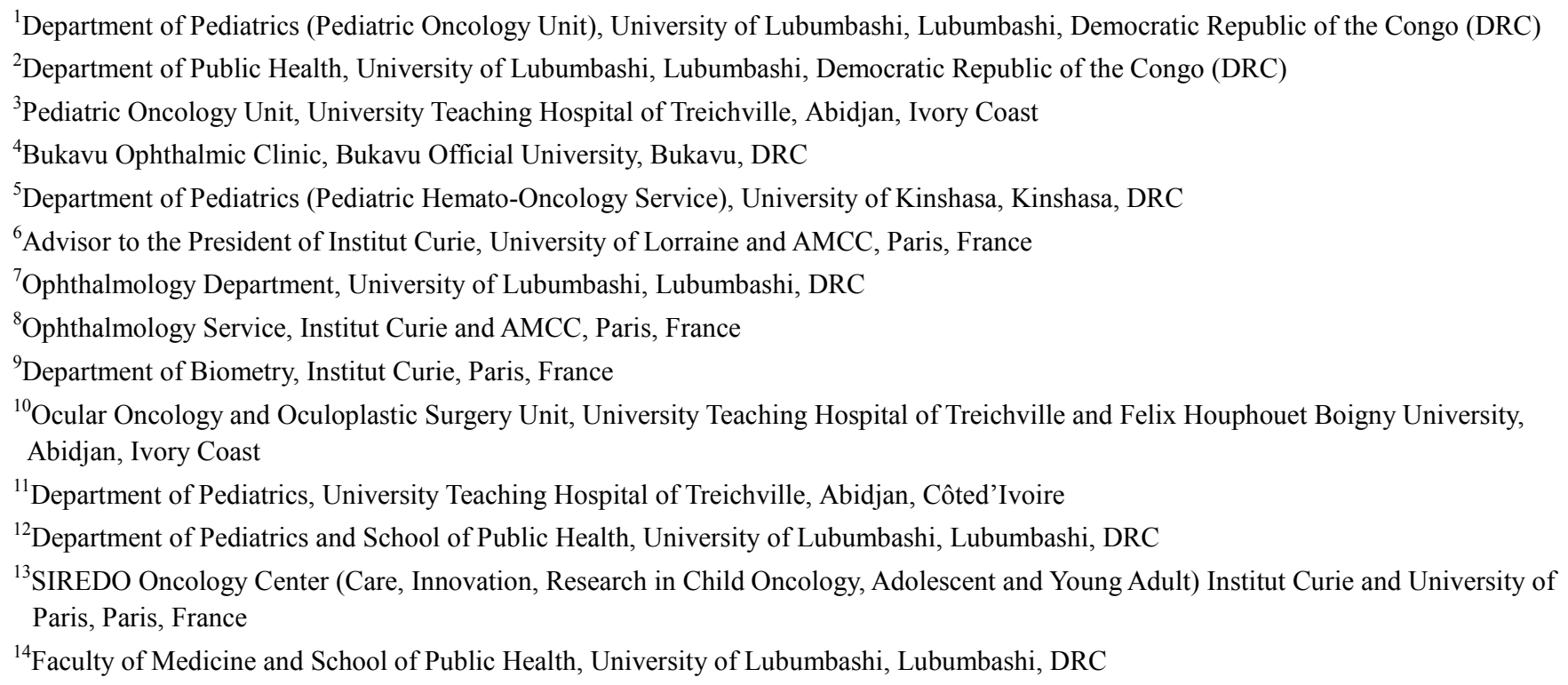

\section{Email address:}

robertlukamba@yahoo.fr (L. M. Robert), mongaben@yahoo.fr (M. B. Ben), attebyjj@yahoo.fr (Y. Atteby), thkabesha@yahoo.com (K. A. Théo), albudiongo@gmail.com (B. N. Aléine), pierrre.bey@curie.fr (B. Pierre), gabybora2003@yahoo.fr (C. B. Gabrielle), laurence.desjardins@curie.fr (D. Laurence), alexia.savignoni@curie.fr (S. Alexia), bereterokia@hotmail.fr (B. Rokia), line.couitchere@gmail.com (C. Line), oscarluboya@yahoo.fr (L. N. Oscar), francois.doz@curie.fr (D. François), Albertmwembotambwe2008@yahoo.fr (M. T. Albert)

${ }^{*}$ Corresponding author

\section{To cite this article:}

Lukamba Mbuli Robert, Monga Bondo Ben, Yao Atteby, Kabesha Amani Théo, Budiongo Nzazi Aléine, Bey Pierre, Chenge Borasisi Gabrielle, Desjardins Laurence, Savignoni Alexia, Berete-Coulibaly Rokia, Couitchere Line, Luboya Numbi Oscar, Doz François, Mwembo Tambwe Albert. Knowledge of Retinoblastoma by Healthcare Professionals in Sub-saharan Africa: Survey Performed in the Republic of Côte d'Ivoire and the Democratic Republic of the Congo. International Journal of Clinical Oncology and Cancer Research.

Vol. 5, No. 2, 2020, pp. 34-38. doi: 10.11648/j.ijcocr.20200502.14

Received: April 25, 2020; Accepted: May 20, 2020; Published: June 15, 2020

\begin{abstract}
Diagnostic delay leads to advanced forms of retinoblastoma, compromising survival. The aim of this study was to evaluate the knowledge of health professionals regarding this disease. This survey was carried out among health professionals in the Democratic Republic of the Congo (cities of Lubumbashi, Bukavu and Kinshasa) and Côte d'Ivoire (Abidjan). Health professionals were divided into 3 professional categories: 1) paediatricians and ophthalmologists, 2) other medical doctors, and 3) paramedics. A questionnaire adapted to each category was used. The response to each question was graded as correct or incorrect. Total knowledge of retinoblastoma for the entire questionnaire was evaluated according to a level of overall score for
\end{abstract}


each health professional: score $<50 \%$ was considered very insufficient, score between 50 and $75 \%$ as insufficient and score $\geq 75 \%$ as sufficient. Descriptive statistical analyses and association measurements were performed. A total of 637 health professionals were surveyed, including 69 in the "pediatricians and ophthalmologists" category, 180 in the "others medical doctors" category and 388 in the "paramedics" category. Globally, more than $90 \%$ of health professionals did not answer all the questions correctly, and $9 \%$ were aware of the most common retinoblastoma-revealing symptoms (leukocoria and strabismus). Overall knowledge of retinoblastoma was sufficient for $26.1 \%$ of Paediatricians and Ophtalmologists, $11.7 \%$ of Other medical doctors and $2.1 \%$ of Paramedicals. One-quarter of health professionals were aware of the two most common signs of retinoblastoma (leukocororia and strabismus) in "pediatricians and ophthalmologists" category, $14 \%$ in "others medical doctors" category and 4\% in "paramedics" category. Most health professionals surveyed in DRC and Cote d'Ivoire were not aware of the retinoblastoma-revealing symptoms. This certainly contributes to delayed diagnosis and the poor prognosis of this disease in those countries.

Keywords: Retinoblastoma, Sub-saharan Africa, Knowledge, Health Professionals

\section{Introduction}

The incidence of retinoblastoma is $1: 15,000$ to 20,000 births per year in the world. This is the most common intraocular malignancy in children. It is the most curable cancer in high-income countries (with more than $95 \%$ healing), thanks in particular to the frequency of early diagnosis, and ease of access to multidisciplinary specialist care $[1,2]$. Early diagnosis is indeed a proven factor of a good prognosis [1]. However, in low-income countries, the prognosis is still bleak, notably due to delayed diagnosis and inadequate management [3, 4].

Lack of knowledge by health professionals of the retinoblastoma revealing symptoms as well as socioeconomic and cultural context contribute to delayed diagnosis [4-7].

Very few studies have evaluated health professionals' knowledge of retinoblastoma [8-10] and, to our knowledge, none has been performed in sub-Saharan Africa: the objective of this work was to evaluate the knowledge of retinoblastoma by health professionals from the Democratic Republic of the Congo (DRC) and the Republic of Côte d'Ivoire.

\section{Methods}

\subsection{Type and Period of the Study}

This work was based on a descriptive cross-sectional study carried out from February $15^{\text {th }}$ to April $15^{\text {th }}, 2015$.

\subsection{Framework of the Study}

This study was conducted with health professionals in three cities of the DRC (Lubumbashi, Bukavu, and Kinshasa) and one city of Côte d'Ivoire (Abidjan). These cities were chosen because the paediatric oncology units of Lubumbashi, Kinshasa, and Abidjan, as well as the Ophthalmological Center of Bukavu, were already involved in a research collaboration network as part of a program for early diagnosis and access to retinoblastoma treatment in subSaharan Africa [11].

\subsection{Population and Sampling}

Convenience sample was carried out in the healthcare professionals' population. Included were health professionals residing in the city who were present on the day of the survey and who agreed to answer the questions. Due to the limited time and the availability of few investigators, out of 475 health facilities repertorieted, we were able to interview only 42 of them. The choice of 42 health facilities took into account:

1. geographic representativeness: according to the distribution of the different health districts.

2. the representativeness of the 3 levels of health facilities; health center, reference hospital, university teaching hospital in each city.

3. the representativeness of health facilities with or without an ophthalmology service.

All categories of health facilities were therefore represented.

A total of 638 health professionals were surveyed on 17692 professionals in the 4 cities concerned (3172 doctors and 14520 paramedics); all the 638 professionals who received a surveyed gave answers.

\subsection{Data Collection}

A self-administered questionnaire was used. The information was collected by trained investigators.

Three types of questionnaires were adapted for 3 categories of health professionals according to their qualifications:

1) Paediatricians (and their residents or interns) and ophthalmologists (and their residents or interns);

2) Other medical doctors: generalists and specialists;

3) Paramedics: Auxiliaries, nurses, executive nurses, and laboratory and imaging technicians.

The investigators passed once to each medical center. After a brief explanation, they gave the questionnaire to the health professional that were required to answer on the spot. It was not allowed that the investigators get back the file after a few hours or the next day. The questions were closed-ended to facilitate data collection.

The main variables studied were: gender, qualification, work experience, work institution, knowledge of signs, 
diagnosis and management of retinoblastoma.

The institutions were grouped into 3 levels:

I: Health center or dispensary;

II. 2nd level hospital;

III. University Teaching Hospital (UTH) or equivalent Hospital.

\subsection{Data Analysis}

The data were entered on epi-info 7.1 software and analyzed using SAS 9.3 software (SAS Institute Inc., Cary, NC, USA). The survey was first tested in a medical facility in Lubumbashi. Each health professional was evaluated according to his/her category. The response to each question was graded as correct or incorrect. Total knowledge of retinoblastoma for the entire questionnaire was evaluated according to a level of overall score for each health professional: score $<50 \%$ was considered very insufficient, score between 50 and $75 \%$ as insufficient and score $\geq 75 \%$ as sufficient.

Results were presented in counts and percentage for qualitative variables, and as position and dispersion parameters for quantitative variables. Bivariate analyzes were carried out to evaluate the relationship between the score obtained and the anthropometric and professional parameters of the interviewees (qualification, institution, professional experience). The Anova test was used to compare these scores according to the categories of the qualitative variables. It was replaced by the non-parametric tests of Wilcoxon-Mann-Whitney and Kruskall-Wallis if the distribution of the quantitative variables was not Gaussian. The link between the quantitative variables (score and professional experience) was done by the Spearman correlation test. The associations of the qualitative variables were explored with the Chi-square or Fischer test if necessary.

To facilitate the comparison of these variables, we grouped the data for some variables whose number was too small: in the first category of health professionals, the institutions were grouped into Hospitals and UTHs.

For all comparisons, the two-sided type one error used was $5 \%$.

\section{Results}

In total 637 professionals were included in the analysis of our study: 249 physicians $(7,8 \%)$ and 388 paramedics $(2$, $7 \%)$.

The sex ratio was 0.97 in the "pediatricians and ophthalmologists category", 2.27 in the "other doctors category" and 0.52 in the "paramedics category".

There were 69 health professionals interviewed in the "pediatricians and ophthalmologists category", 180 in the "other doctors category", and 388 in the "paramedics category" (Table 1).

Table 1. Knowledge of retinoblastoma by health professionals in Démocratic Republic of the Congo (DRC) and Côte d'Ivoire.

\begin{tabular}{|c|c|c|}
\hline I) Ophthalmologists and pediatricians. & $\mathrm{N}=69$ & \\
\hline Questions & Correct & $\%$ \\
\hline 1. Definition of retinoblastoma & 44 & 63.8 \\
\hline 2. The two main revealing symptoms of retinoblastoma & 17 & 24.6 \\
\hline 3. To whom to be referred? & 43 & 62.3 \\
\hline 4. Who decides on the treatment regimen? & 50 & 72.5 \\
\hline 5. Means of treatment of retinoblastoma & 26 & 37,7 \\
\hline Total knowledge & Frequency & $\%$ \\
\hline Very Insufficient & 27 & 39.1 \\
\hline Insufficient & 24 & 34.8 \\
\hline Sufficient & 18 & 26.1 \\
\hline II) Other medical doctors & $\mathrm{N}=180$ & \\
\hline Questions & Correct & $\%$ \\
\hline 1. Definition of retinoblastoma & 85 & 47.5 \\
\hline 2. The two main revealing symptoms of retinoblastoma & 25 & 14 \\
\hline 3. To whom to be referred? & 113 & 62.8 \\
\hline 4. Who treats retinoblastoma? & 94 & 52.2 \\
\hline 5. Retinoblastoma Management Center in the Community & 65 & 36.3 \\
\hline Total knowledge & Frequency & $\%$ \\
\hline Very insufficient & 97 & 53.9 \\
\hline Insufficient & 62 & 34.4 \\
\hline Sufficient & 21 & 11.7 \\
\hline III) Paramedics. & $\mathrm{N}=388$ & \\
\hline Questions & Correct & $\%$ \\
\hline 1. Definition of retinoblastoma & 130 & 33.5 \\
\hline 2. The two main revealing symptoms of retinoblastoma & 15 & 3.9 \\
\hline 3. Who makes the diagnosis? & 264 & 68 \\
\hline 4. Retinoblastoma Management Center in the Community & 90 & 23.2 \\
\hline Total knowledge & frequency & $\%$ \\
\hline
\end{tabular}




\begin{tabular}{lll}
\hline I) Ophthalmologists and pediatricians. & $\mathrm{N}=69$ & \\
Very insufficient & 323 & 83.2 \\
Insufficient & 57 & 14.7 \\
Sufficient & 8 & 2.1 \\
\hline
\end{tabular}

Very insufficient: Score $<50 \%$.

Insufficient: $50 \leq$ Score $<75 \%$.

Sufficient: Score $\geq 75 \%$.

The level of medical facilities was reported for 372 health professionals: 44 professionals worked in first-level structures, 200 in second-level structures, and 128 in thirdlevel structures.

The median work experience was 10 years (Q1-Q3: 6-11) for health professionals in the first category; it was 5 years (Q1-Q3: 2-8.5) for those in the second category and 9 years (Q1-Q3: 5.5-20) for those in the third category.

Regarding the knowledge of health professionals on retinoblastoma by category (Table 1 ), we found that more than $90 \%$ of health professionals did not answer all the questions correctly, and 9\% were aware of the most common retinoblastoma-revealing symptoms (leukocoria and strabismus). Overall knowledge of retinoblastoma was sufficient for $26.1 \%$ of Paediatricians and Ophtalmologists, $11.7 \%$ of Other medical doctors and $2.1 \%$ of Paramedicals. The two man revealing symptoms of retinoblastoma were known by $24.6 \%$ of Paediatricians and Ophtalmologists, $14 \%$ of Other Medical Doctors and 3.9\% of Paramedicals. Comparing the knowledge of retinoblastomas with anthropometric and socio-occupational parameters, we observed that, in the "pediatricians and ophthalmologists category", the means of retinoblastoma treatment (Question 15) were better known by ophthalmologists than by pediatricians $(p=0.03)$. In the "paramedics" category, the global knowledge was associated with the qualification of staff (it was higher among nurses, $p=0.006$ ) and work experience $(\mathrm{p}=0.02)$. Knowledge of retinoblastoma signs was significantly associated with staff qualification $(\mathrm{p}=0.04)$ and work experience $(\mathrm{p}=0.01)$.

\section{Discussion}

In all categories of health professionals, at least $90 \%$ did not answer all questions about the definition, diagnosis, and management of retinoblastoma. In addition, 91\% did not know the two most common retinoblastoma-revealing symptoms: leukocoria and strabismus. Most health professionals were not familiar with retinoblastoma, and especially with signs allowing early diagnosis.

Socio-economic and cultural factors may influence the delayed diagnosis of retinoblastoma [4]. The parents' lack of knowledge of the disease is considered a factor in the delay of the diagnosis [5, 12]. However, the lack of knowledge of this pathology by health professionals has also been mentioned as a factor in the diagnostic delay [7, 12-15]. Assessments of medical students and practitioners have shown that they are not sufficiently aware of the signs when screening for eye conditions in children with retinoblastoma
$[8,9]$. A survey conducted in Mexico showed a clear deficit in knowledge of retinoblastoma by health professionals: only $39.7 \%$ knew the two most common revealing signs of retinoblastoma [10]. Knowledge of these two signs was even lower in our study. This is probably linked to insufficient teaching about the notions of childhood cancer in our university curriculum.

This study may be limited by a selection bias because sampling was not realized at random, but this is not of great concern because the sample was relatively large and all kinds of health professionals were represented. The late diagnosis of retinoblastoma contributes to advanced forms and poor prognosis [3, 16-20]. On the other hand, early diagnosis of retinoblastoma contributes to a better prognosis $[1,3]$. It is therefore imperative to reinforce the knowledge of retinoblastoma among health professionals to improve the prognosis in the DRC and Côte d'Ivoire. We wish that the programme developed by AMCC and his partners to support early diagnosis, access to treatment, and rehabilitation of children with retinoblastoma, in African sub-Saharan countries will achieve this goal [21]

\section{Conclusion}

Most of the Health professionals of DRC and the Republic of Côte d'Ivoire didn't know retinoblastoma diagnosis and therapeutic principles. Most of them were not aware of the most common retinoblastoma-revealing symptoms (leukocoria and strabismus). The knowledge of this pathology was much lower among paramedics and higher among paediatricians and ophthalmologists. Neverless, results were bad for all health professionals. Hence, there is a need for more educational programs on retinoblastoma, especially for the health professionnals awarness on first symptoms of retinoblastoma to improve early diagnosis of this pediatric cancer.

\section{Conflicts of Interest}

The authors declare that they have no competing interests.

\section{Acknowledgements}

We thank the AMCC (Global Alliance Against Cancer), the Institut Curie, and the GFAOP (Franco-African Pediatric Oncology Group) for the realization of the retinoblastoma project in sub-Saharan Africa. We also thank the Sanofi Espoir Foundation and the Pierre Gilles de Gennes 
Foundation for their financial contributions to the development of the project for this study. In particular, we would like to thank Prof. Dr. Jean-Lambert Gini Ehungu of Kinshasa University Clinics, Dr Jean Michon from the Institut Curie, Dr Marcel Numbi and Dr Gray Kanteng from the University Clinics of Lubumbashi for their contributions in carrying out this work.

\section{References}

[1] Aerts I, Lumbroso-Le Rouic L, Gauthier-Villars M, et al. Retinoblastoma update. Arch Pediatr 2016; 23 (1): 112-6.

[2] Singh G \& Daniels AB. Disparities in Retinoblastoma Presentation, Treatment, and Outcomes in Developed and Less-Developed Countries. Seminars in Ophthalmology 2016; 31 (4): 310-6.

[3] Ortiz M V, Dunkel IJ. Retinoblastoma. J Child Neurol. 2016; $31(2): 227-36$

[4] Chantada GL, Qaddoumi I, Canturk S, et al. Strategies to Manage Retinoblastoma in Developing Countries. Pediatr Blood Cancer 2011; 56 (3): 341-8.

[5] Traoré F, Togo B, Sylla F, et al. Retinoblastoma: inventory in Mali and program to develop early diagnosis, treatments and rehabilitation. Bull Cancer 2013; 100 (2): 161-5.

[6] Togo B, Sylla F, Traoré F, et al. A 30-month prospective study on the treatment of retinoblastoma in the Gabriel Toure Teaching Hospital, Bamako, Mali. Br J Ophthalmol. 2010; 94 (4): 467-9.

[7] Goddard AG, Kingston JE, Hungerford JL. Delay in diagnosis of retinoblastoma: risk factors and treatment outcome. $\mathrm{Br} \mathrm{J}$ Ophtamology. 1999; 1320-3.

[8] Rahi JS, Lynn R. A survey of paediatricians ' practice and training in routine infant eye examination. Arch Dis Child. 1998; 78 (4): 364-6.

[9] Saguet P, Lux A, Denion G, et al. Are the Ophthalmology Items of the French Health Record Realistic? Arch Pediatr. 2016; 23 (1): 14-20.

[10] Leal-leal CA, Dilliz-nava H, Flores-rojo M, et al. First Contact
Physicians and Retinoblastoma in Mexico. Pediatr Blood Cancer. 2011; 57 (7): 1109-12.

[11] Howard SC, Zaidi A, Cao X, et al. The My Child Matters programme: effect of public-private partnerships on paediatric cancer care in low-income and middle-income countries. Lancet Oncol 2018; 19 (5): e252-66.

[12] Chantada G, Fandiño A, Manzitti J, et al. Late diagnosis of retinoblastoma in a developing country. Arch Dis Child. 1999; 80 (2): 171-4.

[13] Brown BJ, Adeleye AO, Ibeh JN. A Prospective Study on the Causes of Delayed Diagnosis of Childhood Cancer in Ibadan, Nigeria. Pediatr Hematol Oncol. 2015; 32 (6): 365-73.

[14] Cecen E, Gunes D, Mutafoglu K, et al. The Time to Diagnosis in Childhood Lymphomas and Other Solid Tumors. Pediatr Blood Cancer. 2011; 57 (3): 392-7.

[15] Mattosinho CCS, GrigorovskiN, and Lucena E, et al. Prediagnostic Intervals in Retinoblastoma: Experience at an Oncology Center in Brazil. J Glob Oncol 2016; 3 (4): 323-330.

[16] Waddell KM, Kagame K, Ndamira A, et al. Clinical features and survival among children with retinoblastoma in Uganda. Br J Ophtalmol. 2015; 99 (3): 387-90.

[17] Kaimbo WKD, Mvitu MM, Missotten L. Presenting signs of retinoblastoma in Congolese patients. Bull Soc Belge Ophtalmol. 2002; 283: 37-41.

[18] Ali A A, Elsheikh SM, Elhaj A, et al. Clinical presentation and outcome of retinoblastoma among children treated at the National Cancer Institute (NCI) in Gezira, Sudan: a single Institution experience. Ophthalmic Genet 2011; 32 (2): 122-5.

[19] Kruger M, Reynders D, Omar F, Schoeman J, et al. Retinoblastoma outcome at a single institution in South Africa. S Afr Med J 2014; 104 (12): 859-63.

[20] Carrim ZI, Kajaige J, Bowman RJ, et al. First-year experience of chemotherapy for advanced retinoblastoma in Tanzania: disease profile, outcomes, and challenges in 2008. J Pediatr Ophthalmol Strabismus 2012; 49 (3): 176-83.

[21] Alliance Mondiale Contre le Cancer. Early Diagnosis and Management of Retinoblastoma. https://www.canceramcc.org/english/programs/early-diagnosis-and-managementof-retinoblastoma/.Accessed 2020-03-27. 